Georgian Mathematical Journal

1(1994), No. 3, 243-249

\title{
A SECOND-ORDER NONLINEAR PROBLEM WITH TWO-POINT AND INTEGRAL BOUNDARY CONDITIONS
}

\author{
S. A. BRYKALOV
}

\begin{abstract}
The paper gives sufficient conditions for the existence and nonuniqueness of monotone solutions of a nonlinear ordinary differential equation of the second order subject to two nonlinear boundary conditions one of which is two-point and the other is integral. The proof is based on an existence result for a problem with functional boundary conditions obtained by the author in [6].
\end{abstract}

The present paper is concerned with the theory of nonlinear boundary value problems for equations with ordinary derivatives, see e.g. [1-4], and is closely related to $[5,6]$. We deal here with the solvability of a certain essentially nonlinear second-order problem.

The following notation is used:

$\mathbb{R}$ is the set of all real numbers;

$[a, b]$ denotes a closed interval where a differential equation is considered, $-\infty<a<b<+\infty$;

$C^{0}$ denotes the space of all continuous functions;

$C^{1}$ is the space of all continuously differentiable functions;

$L_{1}$ denotes the space of all Lebesgue measurable functions with integrable absolute value;

$A C$ stands for the space of all absolutely continuous functions;

$C L_{1}^{2}$ is the space of all $x(\cdot) \in C^{1}$ such that $\dot{x}(\cdot) \in A C$.

We consider the existence of monotone solutions of the boundary value problem

$$
\begin{gathered}
\ddot{x}=f(t, x, \dot{x}), \quad t \in[a, b], \\
\omega(x(a), x(b))=0, \\
\int_{a}^{b} \varphi(|\dot{x}(\tau)|) d \tau=g .
\end{gathered}
$$

1991 Mathematics Subject Classification. 34B15, 34K10. 
The solution $x(\cdot) \in C L_{1}^{2}([a, b], \mathbb{R})$ should satisfy equation (1) almost everywhere. Assume that the function $f:[a, b] \times \mathbb{R} \times \mathbb{R} \rightarrow \mathbb{R}$ satisfies the Carathéodory conditions, i.e. $f\left(t, x_{0}, x_{1}\right)$ is measurable in $t$ for any fixed numbers $x_{0}, x_{1}$ and is continuous in $x_{0}, x_{1}$ for almost every fixed $t$. Assume also that $\left|f\left(t, x_{0}, x_{1}\right)\right| \leq M$ for almost all $t$ and all $x_{0}, x_{1}$, the constant $M$ is positive, the number $g \in \mathbb{R}$ is fixed, the functions $\omega: \mathbb{R} \times \mathbb{R} \rightarrow \mathbb{R}$, $\varphi:[0, \infty) \rightarrow \mathbb{R}$ are continuous, $\omega\left(s_{1}, s_{2}\right)$ is nondecreasing in each of the arguments $s_{1}, s_{2}$ and is strictly increasing at least in one of the two arguments, the set of pairs $s_{1}, s_{2}$ that satisfy equality $\omega\left(s_{1}, s_{2}\right)=0$ is nonempty, the function $\varphi(z)$ strictly increases and

$$
\lim _{z \rightarrow+\infty} \varphi(z)=+\infty
$$

For example, if $\varphi(z)=z$ then the boundary condition (3) fixes $L_{1}$-norm of the derivative of the unknown function. And in the case $\varphi(z)=\sqrt{1+z^{2}}$ the equality (3) fixes the length of the curve which is the graph of the solution $x(t), t \in[a, b]$. Let us note also that the equality $x(a)=g_{0}$, where $g_{0} \in \mathbb{R}$ is a number, can be considered as the simplest special case of (2). Thus, the boundary conditions (2), (3) can describe, in particular, a curve with a fixed length emanating from a given initial point.

Denote

$$
A_{\varphi}=\int_{0}^{b-a} \varphi(M \tau) d \tau
$$

Theorem. If $g \geq A_{\varphi}$ then every solution of boundary value problem (1)-(3) is strictly monotone, and there exist at least one increasing and at least one decreasing solutions.

The above theorem was previously announced by the author, cf. Proposition 2 in [5]. The proof will be given below.

Remark 1. If $g<A_{\varphi}$ then problem (1)-(3) may have no monotone solutions. This is the case, for example, if $f \equiv M>0$.

Remark 2. A similar theorem is valid also for an equation with deviating arguments and for condition (3) where $g=g(x(\cdot))$ is a nonlinear functional.

The proof of Theorem employs (and illustrates) the following existence result for a boundary value problem of the form

$$
\begin{gathered}
\ddot{x}(t)=F(x(\cdot))(t), \quad t \in[a, b], \\
B_{0}(x(\cdot))=B_{1}(\dot{x}(\cdot))=0 .
\end{gathered}
$$


The solution $x(\cdot) \in C L_{1}^{2}=C L_{1}^{2}([a, b], \mathbb{R})$ satisfies equation (4) almost everywhere. The mappings

$$
F: C^{1} \rightarrow L_{1}, \quad B_{0}: C L_{1}^{2} \rightarrow \mathbb{R}, \quad B_{1}: A C \rightarrow \mathbb{R}
$$

are assumed to be continuous. Let us fix a closed set of functions $A \subset C L_{1}^{2}$. Denote $A^{(k)}=\left\{x^{(k)}(\cdot): x(\cdot) \in A\right\}$. Assume that the family of functions $A$ satisfies $A^{(2)}=L_{1}$.

Proposition 1. Let $M, N$ be fixed numbers. Consider the following conditions:

a) if $\|x(\cdot)\|_{C^{1}} \leq N$ then $|F(x(\cdot))(t)| \leq M$ for almost all $t$,

b) if $x(\cdot) \in A$ satisfies (5) and $|\ddot{x}(t)| \leq M$ almost everywhere then $\|x(\cdot)\|_{C^{1}} \leq N$,

c) if $x(\cdot) \in A$ and almost everywhere $|\ddot{x}(t)| \leq M$ then there exist a unique number $c_{0} \in \mathbb{R}$ such that

$$
B_{0}\left(x(\cdot)+c_{0}\right)=0, \quad x(\cdot)+c_{0} \in A,
$$

and a unique number $c_{1} \in \mathbb{R}$ that satisfies

$$
B_{1}\left(\dot{x}(\cdot)+c_{1}\right)=0, \quad \dot{x}(\cdot)+c_{1} \in A^{(1)} .
$$

Conditions a), b), c) imply that problem (4), (5) has at least one solution in $A$.

A more general version of Proposition 1 was proven by the author in [6]. We need also the following simple auxiliary result.

Proposition 2. Let the above given assumptions on $\varphi$ hold. If a function $u:[a, b] \rightarrow \mathbb{R}$ satisfies the Lipschitz condition with the coefficient $M$ and vanishes at least at one point then

$$
\int_{a}^{b} \varphi(|u(\tau)|) d \tau \leq \int_{0}^{b-a} \varphi(M \tau) d \tau .
$$

Here the equality holds only for the following four functions

$$
u= \pm M(t-a), \quad u= \pm M(b-t) .
$$

Proof of Proposition 2. Let $u(s)=0$ for some $s \in[a, b]$. Then $|u(t)| \leq$ $M|t-s|$. Thus

$$
\int_{a}^{b} \varphi(|u(\tau)|) d \tau \leq \int_{a}^{b} \varphi(M|\tau-s|) d \tau
$$


Denote the right-hand side of (6) by $\Psi(s)$. We have

$$
\begin{aligned}
\Psi(s)= & \int_{a}^{s} \varphi(M(s-\tau)) d \tau+\int_{s}^{b} \varphi(M(\tau-s)) d \tau= \\
& =\int_{0}^{s-a} \varphi(M \tau) d \tau+\int_{0}^{b-s} \varphi(M \tau) d \tau .
\end{aligned}
$$

And so, the derivative

$$
\frac{d \Psi}{d s}=\varphi(M(s-a))-\varphi(M(b-s))
$$

is negative for $a \leq s<\frac{1}{2}(a+b)$ and positive for $\frac{1}{2}(a+b)<s \leq b$. Consequently, the value $\Psi(a)=\Psi(b)=\int_{0}^{b-a} \varphi(M \tau) d \tau$ is the maximum of $\Psi(s)$ for $s \in[a, b]$, which is attained only at the ends of the interval. The desired inequality is proven. If the right- and left-hand sides of this inequality are equal then $s$ equals either $a$ or $b$, and besides that (6) turns to equality. Taking into account strict monotonicity of $\varphi$ we come to the conclusion that $|u(t)| \leq M|t-s|$ also turns to equality. Thus, either $u= \pm M(t-a)$, or $u= \pm M(b-t)$.

Proof of Theorem. The boundary value problem (1)-(3) is a special case of problem (4), (5). Really, it suffices to assume

$$
\begin{gathered}
F(x(\cdot))(t)=f(t, x(t), \dot{x}(t)), \\
B_{0}(x(\cdot))=\omega(x(a), x(b)), \\
B_{1}(u(\cdot))=\int_{a}^{b} \varphi(|u(\tau)|) d \tau-g .
\end{gathered}
$$

The mappings $F: C^{1} \rightarrow L_{1}, B_{0}: C^{0} \rightarrow \mathbb{R}, B_{1}: C^{0} \rightarrow \mathbb{R}$ are continuous. Denote by $A_{+}$the set of all monotone nondecreasing functions in $C L_{1}^{2}$ and by $A_{-}$the set of all nonincreasing ones. The sets $A_{+}, A_{-}$are closed in $C L_{1}^{2}$, and $A_{+}^{(2)}=A_{-}^{(2)}=L_{1}$. Condition a) of Proposition 1 holds obviously. Let us verify condition b). Let $x(\cdot) \in C L_{1}^{2}$ satisfy (2), (3), and $|\ddot{x}(t)| \leq M$ be true almost everywhere. Since $\varphi(z) \rightarrow+\infty$ as $z \rightarrow+\infty$ there exists a number $r$ that satisfies $\varphi(r)>(b-a)^{-1} g$. If we suppose that $|\dot{x}(t)| \geq r$ for all $t$ then

$$
\int_{a}^{b} \varphi(|\dot{x}(\tau)|) d \tau \geq(b-a) \varphi(r)>g
$$

which contradicts (3). Consequently, $|\dot{x}(s)|<r$ for at least one $s$. Consider some $l_{1}, l_{2}$ such that $\omega\left(l_{1}, l_{2}\right)=0$. Let us show that

$$
\min \left\{l_{1}, l_{2}\right\} \leq x(\sigma) \leq \max \left\{l_{1}, l_{2}\right\}
$$


for some $\sigma$. Really, if (7) does not hold for any $\sigma \in[a, b]$ then due to continuity of $x(t)$ two cases are possible. Either $x(t)>\max \left\{l_{1}, l_{2}\right\}$ for all $t$, or $x(t)<\min \left\{l_{1}, l_{2}\right\}$ for all $t$. Monotonicity of $\omega$ implies that $\omega(x(a), x(b))>$ $\omega\left(l_{1}, l_{2}\right)=0$ in the first case, and $\omega(x(a), x(b))<0$ in the second case. It follows from (2) that neither of the two cases can take place. The existence of the numbers $r, s, \sigma$ named above and the inequality $|\ddot{x}(t)| \leq M$ imply boundedness of $\|x(\cdot)\|_{C^{1}}$. Let us verify now condition c) for $A=A_{+}$and for $A=A_{-}$. The function $x(\cdot)$ being fixed, the number $c_{0}$ is defined uniquely by the equality $B_{0}\left(x(\cdot)+c_{0}\right)=0$ due to the properties of the real function

$$
B_{0}(x(\cdot)+c)=\omega(x(a)+c, x(b)+c)
$$

of the argument $c$. Really, the function is continuous and strictly increasing. It suffices to show that this function takes both positive and negative values. As above, we fix $l_{1}, l_{2}$ for which $\omega\left(l_{1}, l_{2}\right)=0$. Then for $c>\max \left\{l_{1}-\right.$ $\left.x(a), l_{2}-x(b)\right\}$ we have $\omega(x(a)+c, x(b)+c)>\omega\left(l_{1}, l_{2}\right)=0$, and for $c<$ $\min \left\{l_{1}-x(a), l_{2}-x(b)\right\}$ we obtain $\omega(x(a)+c, x(b)+c)<\omega\left(l_{1}, l_{2}\right)=0$. So, the desired properties of the function $B_{0}(x(\cdot)+c)$ are established. We have only to note that $x(\cdot)+c$ for a fixed $c$ is monotone in the same sense as $x(\cdot)$. Consider now $c_{1}$. Assume that $x(\cdot) \in C L_{1}^{2}$ and almost everywhere $|\ddot{x}(t)| \leq M$. Continuity of $\varphi$ implies that the function

$$
\Phi(c)=\int_{a}^{b} \varphi(|\dot{x}(\tau)+c|) d \tau
$$

is also continuous. With the help of Proposition 2 we obtain the following. For $c \in\left(-\infty,-\max _{t} \dot{x}(t)\right]$ we have $\dot{x}(t)+c \leq 0$, and the function $\Phi(c)$ strictly decreases taking values from $+\infty$ to a number not larger than $A_{\varphi}$; and if $c \in\left[-\min _{t} \dot{x}(t),+\infty\right)$ then $\dot{x}(t)+c \geq 0$, and the function $\Phi(c)$ strictly increases taking values from a number not larger than $A_{\varphi}$ to $+\infty$. A conclusion follows that if $x(\cdot) \in C L_{1}^{2}$ and almost everywhere $|\ddot{x}(t)| \leq M$ then there exists a unique $c_{1}$ that satisfies

$$
B_{1}\left(\dot{x}(\cdot)+c_{1}\right)=0, \quad \dot{x}(t)+c_{1} \geq 0 .
$$

Similarly, conditions

$$
B_{1}\left(\dot{x}(\cdot)+c_{1}\right)=0, \quad \dot{x}(t)+c_{1} \leq 0
$$

also define a unique $c_{1}$. Thus, condition c) is valid. It follows from Proposition 1 that boundary value problem (1)-(3) is solvable in $A_{+}$and in $A_{-}$. Now we have to show that every solution $x(t)$ of this problem is strictly monotone. If the derivative $\dot{x}(t)$ does not vanish then all its values have the same sign, and so $x(t)$ is obviously monotone. Let now the derivative $\dot{x}(t)$ 
vanish at least at one point. Using Proposition 2 we obtain

$$
\int_{a}^{b} \varphi(|\dot{x}(\tau)|) d \tau \leq A_{\varphi}
$$

Taking into account the inequality $A_{\varphi} \leq g$ and boundary condition (3) we see that the two values in (8) are equal. Employing again Proposition 2 we conclude that either $\dot{x}(t)= \pm M(t-a)$, or $\dot{x}(t)= \pm M(b-t)$. And since $M \neq 0$ the function $x(t)$ is strictly monotone. Theorem is proven. Let us note that Theorem can be proven also basing on results of [7].

In conclusion we verify Remark 1 . Assume $f \equiv M>0, g<A_{\varphi}$. We need to show that problem (1)-(3) has no monotone solutions. The equation (1) takes the form $\ddot{x}=M$. And since $M \neq 0$ we obtain $\dot{x}(t)=M(t-\gamma)$. Thus, $x(t)$ can be monotone only if $\gamma \leq a$ or $\gamma \geq b$. Let us consider these two cases separately. If $\gamma \leq a$ then $|\dot{x}(\tau)|=M(\tau-\gamma) \geq M(\tau-a)$ for $\tau \in[a, b]$, and (3) implies $g \geq \int_{a}^{b} \varphi(M(\tau-a)) d \tau=A_{\varphi}>g$. This contradiction shows that the inequality $\gamma \leq a$ does not hold. Similarly, if $\gamma \geq b$ then $|\dot{x}(\tau)|=M(\gamma-\tau) \geq M(b-\tau)$ for $\tau \in[a, b]$ and thus $g \geq \int_{a}^{b} \varphi(M(b-\tau)) d \tau=A_{\varphi}>g$. And so, the case $\gamma \geq b$ is not possible either. Remark 1 is verified.

\section{REFERENCES}

1. N.V.Azbelev, V.P.Maksimov and L.F.Rakhmatullina, Introduction to the theory of functional-differential equations. (Russian) "Nauka", Moscow, 1991.

2. A.Granas, R.B.Guenther and J.W.Lee, Some general existence principles in the Carathéodory theory of nonlinear differential systems. J. Math. Pures Appl. 70(1991), no. 2, 153-196.

3. I.T.Kiguradze, Boundary value problems for systems of ordinary differential equations. (Russian) Current problems in mathematics. Newest results, vol. 30, 3-103, Itogi Nauki i tekhniki. Acad. Nauk SSSR, Vsesoyuzn. Inst. Nauchn. i Techn. Inform., Moscow, 1987. English transl. in J. Soviet Math. 43(1988), no. 2, 2259-2339.

4. I.T.Kiguradze and B.L.Shekhter, Singular boundary value problems for second-order ordinary differential equations. (Russian) Current problems in mathematics. Newest results, vol. 30, 105-201, English transl. in J. Soviet Math. 43(1988), No. 2, 2340-2417.

5. S.A.Brykalov, Existence and nonuniqueness of solutions of some nonlinear boundary value problems. (Russian) Dokl. Akad. Nauk. SSSR 
316(1991), no. 1, 18-21. English transl. in Soviet Math. Dokl. 43(1991), No. 1, 9-12.

6. S.A.Brykalov, Solvability of a nonlinear boundary value problem in a fixed set of functions. Differentsial'nye Uravneniya 27(1991), No. 12, 2027-2033. (Russian); English transl. in Differential Equations 27(1991), No. $12,1415-1420$.

7. S.A.Brykalov, Problems with monotone nonlinear boundary conditions. (Russian) Dokl. Akad. Nauk 325(1992), No. 5, 897-900.

(Received 03.05.1993)

Author's address:

Institute of Mathematics and Mechanics

Ural Branch of Russian Academy of Sciences

ul. Kovalevskoi 16

620066 Ekaterinburg

Russia 